This item was submitted to Loughborough's Research Repository by the author.

Items in Figshare are protected by copyright, with all rights reserved, unless otherwise indicated.

\title{
Alginate nanofibrous mats with adjustable degradation rate for regenerative medicine
}

PLEASE CITE THE PUBLISHED VERSION

http://dx.doi.org/10.1021/bm501834m

PUBLISHER

(C) American Chemical Society

VERSION

AM (Accepted Manuscript)

\section{PUBLISHER STATEMENT}

This work is made available according to the conditions of the Creative Commons Attribution-NonCommercialNoDerivatives 4.0 International (CC BY-NC-ND 4.0) licence. Full details of this licence are available at: https://creativecommons.org/licenses/by-nc-nd/4.0/

\section{LICENCE}

CC BY-NC-ND 4.0

\section{REPOSITORY RECORD}

Hajiali, Hadi, Jose A. Heredia-Guerrero, loannis Liakos, Athanassia Athanassiou, and Elisa Mele. 2015. "Alginate Nanofibrous Mats with Adjustable Degradation Rate for Regenerative Medicine". figshare. https://hdl.handle.net/2134/17575. 


\section{Alginate nanofibrous mats with adjustable degradation rate for regenerative medicine}

Hadi Hajiali, ${ }^{*}+, \neq{ }^{\ddagger}$ José A. Heredia-Guerrero, $^{\dagger}$ Ioannis Liakos, ${ }^{\dagger}$ Athanassia Athanassiou, ${ }^{\dagger}$ Elisa Mele $e^{*,+\S}$

$\uparrow^{\dagger}$ Smart Materials, Nanophysics, Istituto Italiano di Tecnologia (IIT), via Morego 30, 16163 Genoa, Italy.

${ }^{\ddagger}$ DIBRIS, University of Genoa, via Opera Pia 13, 16145, Genoa, Italy.

$\S$ Present address: Department of Materials, Loughborough University, Loughborough, Leicestershire, LE11 3TU. E-mail: E.Mele3@lboro.ac.uk.

KEYWORDS: alginate, nanofibres, controlled biodegradation

ABSTRACT: The broad utilization of electrospun scaffolds of sodium alginate in tissue engineering is strongly limited by their high solubility in aqueous environments and by the difficulty to adjust their degradation dynamics. Here, an alternative strategy to enhance the stability and to control the degradability of alginate nanofibres is described by treating them with trifluoroacetic acid for specific time intervals. It is demonstrated that by increasing the duration of the acid treatment procedure, lower degradation rate of the resulting fibres in buffer solutions can be achieved. Furthermore, the produced mats are free from cytotoxic compounds and highly biocompatible. The properties conferred to the alginate nanofibrous mats by the proposed method are extremely attractive in the production of innovative biomedical devices. 


\section{INTRODUCTION}

The tendency to use natural polymers in the biomedical field has increased significantly in recent years ${ }^{1}$. Within this class of biomaterials, alginate, a polysaccharide derived from brown algae, exhibits excellent biocompatibility, low toxicity, non-immunogenicity, and relatively low cost ${ }^{2}$. It finds applications in tissue engineering for $\operatorname{skin}^{3}$, nerve ${ }^{4}$, bone ${ }^{5}$, and cartilage regeneration ${ }^{6}$, and in drug delivery systems ${ }^{7}$. Depending on the different uses, a variety of sodium alginate (SA) structures, such as films ${ }^{8}$, particles ${ }^{9}$, sponges ${ }^{10}$, or fibres ${ }^{11}$, have been proposed. Among these, nanofibrous matrices, mainly produced by the electrospinning technique, are particularly attractive in regenerative medicine, because they mimic the organisation of the native tissue ${ }^{12}$, and possess high porosity for the transport of nutrients, and tuneable mechanical properties ${ }^{13}$. The availability of nanofibrous scaffolds whose biodegradation rate can be modulated is of strategic importance in tissue engineering ${ }^{14}$. Due to the high water solubility of sodium alginate, the corresponding nanofibres are poorly stable in aqueous environments, and consequently their applicability in biomedical sectors is strongly limited. For this reason, different cross-linking approaches have been proposed ${ }^{15}$, including glutaraldehyde (GTA) ${ }^{16}$ and divalent ions ${ }^{17}$. However, some of these methods present drawbacks related to the generation of toxic byproducts during the degradation of the nanofibres in the body ${ }^{18}$, and to the morphological changes of the fibrous meshes under the cross-linking conditions. In particular, it has been demonstrated that GTA reduces the biocompatibility of the cross-linked materials, resulting in cytotoxicity and cell apoptosis ${ }^{19}$. On the other hand, the use of calcium ions for the cross-linking process of the SA nanofibres can affect the morphology of the mats, with the partial or complete loss of their network organisation ${ }^{17 d}$. Most considerably, these cross-linking procedures hardly 
succeed to control and adjust the degradation rate of the fibres, as required for the majority of tissue engineering applications ${ }^{20}$.

Here, we show a simple method to increase and fine adjust the stability of sodium alginate electrospun nanofibres in aqueous environments under physiological conditions. Continuous and uniform nanofibres were fabricated by blending alginate with polyethylene oxide (PEO). The latter is conventionally used to enhance the electro-spinnability of natural polymers, including alginate solutions ${ }^{11,21}$. The chemical structure of the produced nanofibres was properly modified by acidification of carboxylate groups and formation of poly(alginic acid) using trifluoroacetic acid (TFA). We demonstrate that increasing the duration of the TFA treatment procedure from 3 to $24 \mathrm{~h}$, the degradability of the treated nanofibres is extended from 7 to 14 days, while their morphology and network organisation remain intact. Moreover, biocompatibility assays proved that the treated scaffolds are non-cytotoxic and can promote cell attachment and proliferation, thanks to their nano-topography and high specific surface area that mimic the extracellular matrix environment. The combination of adjustable degradation and high biocompatibility makes the treated alginate mats promising as scaffolds for biomedical applications, particularly for wound healing where degradation rates of some days are required.

\section{EXPERIMENTAL METHODS}

\section{Materials and cell line}

Sodium alginate, polyethylene oxide (PEO), Pluronic F127, dimethylformamide (DMF), trifluoroacetic acid (TFA), acetic acid, formic acid, hydrochloric acid ( $\mathrm{HCl})$, sodium hydroxide $(\mathrm{NaOH})$, Dulbecco's modified eagle medium (DMEM), bovine calf serum (BCS), trypsin-EDTA solution, penicillin-streptomycin, phosphate buffered saline (PBS), fluoroshield with DAPI, and 
thiazolyl blue tetrazolium bromide (MTT) were purchased from Sigma Aldrich. Alexa Fluor ${ }^{\circledR}$ 647 Phalloidin was purchased from Life Technology. The NIH/3T3 cell line was obtained from $\operatorname{ATCC}^{\circledR}\left(\mathrm{CRL}-1658^{\mathrm{TM}}\right)$.

\section{Fabrication and stabilisation treatment of the alginate-based electrospun scaffolds}

Aqueous solutions of SA (4 wt $\%)$ and PEO (4 wt $\%)$ were prepared by dissolving separately the two polymers in distilled water. Then, the two solutions were mixed in order to have a SA/PEO volume ratio of $8: 2$. In the final SA/PEO solution, $5 \% \mathrm{v} / \mathrm{v}$ of DMF and $1.5 \% \mathrm{w} / \mathrm{v}$ of Pluronic F127 were added. For the electrospinning process, a syringe with a stainless-steel 23-gauge needle was filled with the final solution and connected to a syringe pump (NE-1000, New Era Pump Systems, Inc.) working at a constant flow rate of $0.5 \mathrm{~mL} / \mathrm{h}$. A voltage of $25 \mathrm{kV}$ was applied between the needle and an aluminium collector (air gap distance of $20 \mathrm{~cm}$ ) by using a high-voltage power supply (EH40R2.5, Glassman High Voltage, Inc.). All the electrospun SA/PEO fibres were vacuum-dried at room temperature.

After electrospinning, the alginate-based nanofibrous mats were mechanically removed from the aluminium collector, and soaked in TFA (99\% pure) for different time intervals $(3,6,12$, and 24 h), in closed containers without agitation and at room temperature. Subsequently, the samples were removed from the acid environment, and vacuum-dried. For sake of comparison, we treated the fibres also with other acids: acetic acid, formic acid and $\mathrm{HCl}$. In particular, the fibrous mat was immersed for $3 \mathrm{~h}$ in acetic acid and formic acid, whereas the treatment with $\mathrm{HCl}$ at $37 \%$ lasted only few minutes due to the complete dissolution of the fibres. A basic solution $(\mathrm{NaOH}$ $0.1 \mathrm{M}$ ) was instead used to solubilise the fibres after the TFA procedure. 


\section{Characterisation of the electrospun mats}

The morphology and the size distribution of the electrospun alginate-based fibres, before and after the TFA treatment, were analysed by scanning electron microscopy (SEM). A JEOL JSM6490LA microscope working in high vacuum mode was used. Prior to observation, a thin film of gold $(5 \mathrm{~nm})$ was deposited onto the samples by ion sputtering. The SEM images were processed by Image J and Adobe Photoshop to measure the average diameter of the nanofibres (50 nanofibres were analysed) and the total surface area of the electrospun mats, respectively.

The chemical analysis of SA, PEO, and the SA/PEO nanofibrous mats, before and after the acid treatment, was performed by an attenuated total reflectance (ATR) accessory (MIRacle ATR, PIKE Technologies) coupled to a Fourier transform infrared (FTIR) spectrometer (Equinox 70

FT-IR, Bruker). All spectra were recorded over a range of 4000-600 $\mathrm{cm}^{-1}$, with $4 \mathrm{~cm}^{-1}$ resolution (accumulating 32 scans). Additionally, the elemental composition of the pristine and treated (24 $\mathrm{h}$ in TFA) alginate-based nanofibres was revealed by X-ray photoelectron spectroscopy (XPS) using a Mg anode X-ray source (SPECS XRC-1000 from SPECS LAB GmbH, Berlin) set at $1253.6 \mathrm{eV}$ and a Phoibos analyzer Has 3500 (Hemispherical Energy Analyzer).

\section{Degradation study}

The in-vitro degradation of the samples was carried out in a phosphate buffered saline (PBS) solution at $\mathrm{pH} 7.4$, in three replicates. All samples were placed individually in sealed bottles containing PBS and kept in a water bath at $37^{\circ} \mathrm{C}$. At time periods of 1 week, the PBS solutions were replaced with fresh ones. After 1, 4, 7, 10, and 14 days, the samples were removed from the PBS, washed thoroughly with distilled water, and then vacuum-dried to measure their weight loss. The weight loss percentage was calculated as follows ${ }^{22}$ :

Percentage of weight loss $=[($ final weight - initial weight $) /$ initial weight $] \times 100$ 


\section{Biocompatibility study}

Fibroblast cells (NIH/3T3-cells) were used to investigate the biocompatibility of the TFA treated alginate-based nanofibres. They were cultured in DMEM supplemented with $10 \%$ of BCS and $1 \%$ of antibiotics $\left(100 \mathrm{U} / \mathrm{mL}\right.$ penicillin and $0.1 \mathrm{mg} / \mathrm{mL}$ streptomycin) in an incubator at $37{ }^{\circ} \mathrm{C}$ with $5 \% \mathrm{CO}_{2}$. The culture medium was replaced every 3 days. First, the nanofibrous scaffolds were sterilized under ultraviolet (UV) light for $30 \mathrm{~min}$, and then washed using a PBS solution. Before seeding the cells on the nanofibres, the fibroblasts were detached from the culture flask using trypsin/EDTA solution, when they were grown to nearly $80 \%$ confluence. As control, cells were seeded at the same density on glass cover slips.

In order to determine the proliferation rate on the scaffolds, the cells were cultured for 5 days. The percentage of adhered and proliferated cells was measured after 1,3 , and 5 days using a colorimetric MTT assay. Furthermore, the morphology of cells seeded for 1 day on the nanofibrous mats treated with TFA for $24 \mathrm{~h}$ was analysed. The samples were fixed in $3.7 \%$ formaldehyde for $15 \mathrm{~min}$ before rinsing with PBS for 3 times. The samples were then permeabilised with $1 \%$ Triton X-100, and stained with alexa-fluor 647 phalloidin and DAPI for actin cytoskeleton and nucleus, respectively. Cell imaging was then carried out by the confocal microscope Nikon A1.

\section{Statistical analysis}

All the measured values were expressed as mean \pm standard deviation (SD). The data were analyzed by the Student's t-test, and differences were considered significant at $\mathrm{P}<0.05$. 


\section{RESULTS}

\section{Fabrication of the alginate-based electrospun nanofibres}

As shown in Figure 1A, we were able to electrospin alginate-based nanofibres that were free from beads and defects, and exhibited cylindrical shape and regular morphology, with an average diameter of $(90 \pm 20) \mathrm{nm}$. As expected, the as-prepared nanofibres were highly water soluble, and dissolved easily in water-based media or even in humid environments. In order to overcome this drawback and to improve the stability in water of the nanofibres, we developed a novel strategy based on the use of Trifluoroacetic acid. In particular, the SA/PEO electrospun mats were immersed in pure TFA for time periods ranging from 3 to $24 \mathrm{~h}$. The acid procedure induced a chemical modification of the nanofibres' structure (as will be described in discussion) while maintaining their shape and network organisation (SEM analysis in Figure 1). From the comparison between the as-prepared (Fig. 1A) and treated samples (Fig. 1B-1E), it can be noted that the layers of fibres become more packed after soaking in the acidic medium. Consequently, after the treatment the total surface area of the mat occupied by the fibres $\left(\mathrm{A}_{\text {fibres }}\right)$ increased from $60 \%$ (untreated samples) to $75 \%$ (treated samples). After 3 or $6 \mathrm{~h}$ in TFA (Fig. 1B and 1C, respectively), $A_{\text {fibres }}$ was $75 \%$, whereas it was about $80 \%$ when the treatment was extended up to 12 or $24 \mathrm{~h}$ (Fig. 1D and 1E, respectively). The fibres' diameter slightly increased after the acid procedure, reaching a final value of around $100 \mathrm{~nm}$ after $24 \mathrm{~h}$ in TFA. Nevertheless, the pores of the treated electrospun mats were still open, without the presence of large flatten and unstructured regions. Therefore, the developed TFA treatment had a negligible effect on the morphology of the electrospun fibrous mats that maintained their mechanical flexibility, since they could be easily handled even after $24 \mathrm{~h}$ in TFA (Figure 1F). 


\section{Chemical characterisation of the fibres after the TFA treatment}

The effect of the TFA process on the chemistry of the produced alginate-based nanofibres was analysed by ATR-FTIR and XPS. In Figure 2A, bands associated with polyethylene oxide and sodium alginate were easily distinguishable in the spectrum of the SA-PEO fibres. Absorption bands assigned to PEO were: $\mathrm{CH}_{2}$ scissoring at $1466 \mathrm{~cm}^{-1}, \mathrm{CH}_{2}$ wagging at 1359 and $1342 \mathrm{~cm}^{-1}$, $\mathrm{CH}_{2}$ twisting at $1278 \mathrm{~cm}^{-1}, \mathrm{C}-\mathrm{O}-\mathrm{C}$ stretching at $1095 \mathrm{~cm}^{-1}, \mathrm{CH}_{2}$ rocking at 960 and $841 \mathrm{~cm}^{-1} 23$. Also, some bands ascribed to sodium alginate were evident: asymmetric and symmetric $\mathrm{COO}^{-}$ stretching vibrations at 1604 and $1412 \mathrm{~cm}^{-1}$, respectively, and the asymmetrical C-O-C stretching modes at $1029 \mathrm{~cm}^{-1} 24$. Infrared spectra of pure components, PEO and SA, are shown in Figure S1. All infrared bands of the SA-PEO nanofibres had the same position and relative intensity of the pure components. Only two exceptions were observed: the position of the asymmetric $\mathrm{COO}^{-}$ stretching band was shifted from $1595 \mathrm{~cm}^{-1}$ for the pure SA to $1604 \mathrm{~cm}^{-1}$ for the SA-PEO fibres, and the peak of the $\mathrm{O}-\mathrm{H}$ stretching vibration shifted from $3267 \mathrm{~cm}^{-1}$ for SA to $3352 \mathrm{~cm}^{-1}$ for SAPEO nanofibres (Figure S2).

After the TFA treatment changes in the ATR-FTIR spectrum of the fibres were noticed. Firstly, absorption peaks of PEO (see above) and TFA (i.e., bands associated with the trifluoromethyl or carbonyl groups of the acid) were not detected, indicating that the PEO was solved by the acid and that the removal of TFA was complete. Secondly, a new doublet band ca. $1725 \mathrm{~cm}^{-1}$ and $1632 \mathrm{~cm}^{-1}$ appeared that is related to the $\mathrm{COOH}$ stretching of free carboxylic acid groups in alginic acid structures (inset of Figure 2B) ${ }^{24 b}$. At the same time, bands ascribed to carboxylate groups disappeared. The ratio between the intensities of the $\mathrm{COOH}$ stretching band $\left(1725 \mathrm{~cm}^{-1}\right)$, related to the acid treatment, and the asymmetric C-O-C stretching band $\left(1029 \mathrm{~cm}^{-1}\right)$, which instead is not affected by the TFA treatment, was monitored for the different time intervals of the 
treatment to estimate the presence of carboxylic acid in the nanofibres (Figure 2B). This ratio was rapidly increased in the initial stages of the acid treatment (up to $6 \mathrm{~h}$ ) and reached a constant value afterwards. Furthermore, we analysed the ratio between the intensity of the bands at 808 and $787 \mathrm{~cm}^{-1}$ (Figure 2C) that gives a semi-quantitative estimation of the composition of alginate in terms of percentage of mannuronic $(M)$ and guluronic $(G)$ units $\left(M / G\right.$ ratio) ${ }^{25}$. We noticed that the $\mathrm{M} / \mathrm{G}$ ratio decreased by increasing the time of the TFA treatment, indicating the prevalence of $G$ units in the alginate nanofibres.

XPS spectra were acquired to further characterise the chemical properties of the electrospun nanofibres and the effect of the acid treatment. Table S1 in the Supporting Information shows the atomic composition of the nanofibres before and after the TFA treatment $(24 \mathrm{~h})$. A reduction of the percentage of $\mathrm{C}$ (from 69 to $59 \%$ ) and $\mathrm{Na}$ (from 0.7 to $0 \%$ ) was observed after the immersion in the acid, while the percentage of $\mathrm{O}$ increased (from 30.3 to $41.0 \%$ ). In both cases, $\mathrm{F}$ was not detected, indicating the complete removal of TFA from the samples, in good agreement with the ATR-FTIR results. In addition, high-resolution XPS spectra of the fibres in the C1s energy range were acquired (Figure 3). The C1s core-level spectrum of the SA-PEO fibres was curve-fitted in four components at $284.9,286.5,288.0$, and $289.9 \mathrm{eV}$ corresponding to $\mathrm{CC} / \mathrm{CH}, \mathrm{COH} / \mathrm{COC}$, OCO, and COO, respectively. The second component $(\mathrm{COH} / \mathrm{COC})$ arises from both $\mathrm{PEO}$ and SA, whereas the last two components (OCO and $\mathrm{COO}$ ) arise from SA molecules. After $24 \mathrm{~h}$ of acid treatment, main differences were associated with a reduction of the $\mathrm{COH} / \mathrm{COC}$ area from 72 to $56 \%$, and to an increment of the $\mathrm{COO}$ and $\mathrm{OCO}$ areas from around 2 to $15 \%$. These changes in the chemical composition of the nanofibres can be related to the dissolution of PEO (rich in COC groups) by TFA, as previously discussed for the infrared analysis. 


\section{Degradability analysis of the fibrous scaffolds under physiological conditions}

The degradation of the alginate nanofibres in an aqueous environment, before and after the TFA treatment, was studied by incubating the samples in PBS, and measuring their weight loss after different time intervals (Figure 4). As expected, the alginate fibres that were not exposed to TFA dissolved completely after 1 day. On the contrary, we observed a slower degradation for the TFA-treated samples, and we were able to adjust it by changing the duration of the acid treatment. For instance, the nanofibrous mats that were in TFA for $3 \mathrm{~h}$ exhibited a weight loss of $50 \%$ during the first day of PBS incubation, and a complete dissolution within 10 days. By increasing the duration of the TFA treatment up to $6 \mathrm{~h}$, we measured a weight reduction of $37 \%$ after one day, and it exceeded 50\% only after 7 days. Furthermore, the fibres were still resisting in the PBS medium for more than 10 days. When the TFA procedure was extended up to 12 and $24 \mathrm{~h}$, the degradation time of the electrospun samples was of 14 days, with a high stability in the first 7 days (weight loss of around 43\%).

SEM investigations of the treated fibrous scaffolds after immersion in the aqueous medium for one week confirmed the weight loss results (Figure S3). After 3 (Figure S3A) or 6 h (Figure S3B) of the TFA procedure, a marked deterioration of the electrospun mat was observed, even if open pores and fibrillar structures were still visible. Instead, most of the fibres were intact and preserved their morphology, when the TFA treatment was of 12 (Figure S3C) or 24 h (Figure S3D).

\section{Biocompatibility assays}

The biocompatibility of the TFA-treated alginate nanofibres was evaluated using fibroblast cells as model. MTT assays after 1 (Figure 5A), 3 (Figure 5B) and 5 (Figure 5C) days of cell culture revealed that the treated fibrous scaffolds were highly biocompatible, without statistically 
significant differences $(\mathrm{P}>0.05)$ in the fibroblast viability for the analysed samples in comparison with the control (glass substrates). Therefore, cytotoxic compounds were not contained into or released from the electrospun mats. The MTT results were confirmed also by the morphological analysis of the cells. In Figure 5D and 5E, fluorescent micrographs of fibroblast cells cultured for 1 day on the alginate nanofibres (after $24 \mathrm{~h}$ in TFA) were reported. It is clearly visible that the cells were able to attach on the surface of nanofibres, assuming an elongated and flattened morphology (Figure 5E).

\section{DISCUSSION}

Here we propose a method to enhance the stability in water of electrospun alginate nanofibres, allowing the fabrication of scaffolds whose degradation profile can be easily tuned from 7 to 14 days. Our approach is based on the treatment of alginate-PEO nanofibres with trifluoroacetic acid for specific time intervals. In this way, significant changes in the chemical structure of the fibres are induced.

The comparison between the FTIR spectrum of pure sodium alginate and that one of SA-PEO nanofibres before the TFA treatment (Figure S1 and S2) shows two main differences in the position of the asymmetric $\mathrm{COO}^{-}$stretching band and $\mathrm{O}-\mathrm{H}$ stretching band. In similar SA-PEO systems, these shifts to higher wavenumbers are related to the preferential formation of hydrogen bonds between the ether (C-O-C) group of the PEO chains and the hydroxyl groups of alginate, partially excluding the carboxylate groups of such interactions ${ }^{26}$. After the acid treatment, it was observed that the TFA has a double effect: the protonation of carboxylate groups, and the solubilisation of some components of the fibres. The protonation can be justified by an acid-base reaction between carboxylate groups from alginate and TFA molecules, since the $\mathrm{pK}_{\mathrm{a}}$ of alginate 
$\mathrm{COO}^{-}$is around 10.5 and around 0.5 for TFA ${ }^{27}$. In these conditions, TFA molecules can transfer protons to the $\mathrm{COO}^{-}$groups, forming the corresponding salt (sodium trifluoroacetate, $\mathrm{NaOOCCF}_{3}$ ) and carboxylic acid functional groups [poly(alginic acid)]. The reaction is fast in the initial stages, and reaches the thermodynamic equilibrium after $12 \mathrm{~h}$ (Figure 2B). Moreover, the disappearance of the XPS peak ascribed to $\mathrm{Na}$ after $24 \mathrm{~h}$ of acid treatment is indicative of a practically full conversion of carboxylate groups into carboxylic acids. On the other hand, TFA acts as a solvent, since FTIR and XPS investigations indicate that no PEO and $\mathrm{NaOOCCF}_{3}$ exist in the TFA-treated fibres, and the $\mathrm{M} / \mathrm{G}$ ratio decreases as well (Figure $2 \mathrm{C}$ ). The removal of PEO and of the produced salt offers us the possibility to obtain nanofibres of pure alginate, whose chemical properties can be actively exploited for cellular interactions ${ }^{20}$. On the other hand, the changes of the $\mathrm{M} / \mathrm{G}$ ratio of the nanofibres could be caused by the different solubility of the homopolymer blocks of the alginic chains in the acid medium (higher for homopolymannuronic blocks and lower for homopolyguluronic blocks) ${ }^{28}$. Figure 6 shows a schematic representation of the role played by TFA on the chemical structure of the alginate fibres.

The nanofibres treated by TFA can be quickly solubilised by basic solutions $(\mathrm{NaOH})$. The ATRFTIR spectrum of the residues shows strong absorptions associated with carboxylate groups together with vibrations ascribed to $-\mathrm{COOH}$ functional groups interacting by H-bonds (Figure S4). This is an indication that in this way free carboxylic acid groups (typical of the polyalginic fibres) can be converted back to carboxylate groups (typical of pure alginate). Therefore, chemical changes on the alginate/polyalginic acid system can be induced by acid-base reactions. Interestingly, we are able to control the biodegradation rate of the electrospun constructs by tuning the duration of the TFA treatment (from 3 to $24 \mathrm{~h}$ ). In particular, the stability of the treated nanofibres in aqueous media is directly related to the amount of poly(alginic acid) 
produced during the TFA process. In fact, it is well known that alginic acid is insoluble in water ${ }^{29}$ and it has low water sorption ${ }^{29 b, 30}$. As shown in Figure $2 \mathrm{~B}$, the ratio $v_{\mathrm{COOH}} / v_{\mathrm{aC}-\mathrm{O}-\mathrm{C}}$, which can be considered an indication of the presence of alginic acid in the nanofibres, is maximised for 12 or $24 \mathrm{~h}$ of acid treatment. Indeed, the electrospun mats treated for 12 or $24 \mathrm{~h}$ with TFA are stable in water for 14 days, differently from the other samples that degrade in 10 days or less. Hence, the stability of the nanofibres in aqueous environments increases with the time of the treatment in TFA. A direct comparison between our method and others proposed to cross-link alginate nanofibres shows that in the first case the degradation of the scaffolds in aqueous solutions is much better controlled. For instance, SA fibres cross-linked by $\mathrm{Ca}$ ions can lose more than $60 \%$ of their weight after 1 day in normal saline solutions ${ }^{31}$ and deionized water ${ }^{20}$, whereas in our case the loss can be reduced to $35 \%$.

A main aspect to point out is that the morphology of the nanofibres and their network organisation is preserved during the acid treatment, overcoming one of the main limitations of other approaches that have been proposed to cross-link sodium alginate nanofibres ${ }^{17 \mathrm{~d}}$. In our case, after the treatment, changes in the size of the elecrospun fibres are negligible, and the interconnected porosity and the high exposed surface area is maintained (Figure 1). TFA possesses the unique ability to induce the discussed chemical modifications on the sodium alginate fibres, without affecting their morphology. In fact, the treatment of the SA-PEO electrospun mats with other acids (acetic acid, formic acid and $\mathrm{HCl}$ ) unlikely results in an enhancement of the stability of the fibres and most commonly determines a deterioration of the fibrous network. For instance, after immersion in acetic acid $\left(\mathrm{pK}_{\mathrm{a}}=4.8\right)$, the ATR-FTIR spectrum of the nanofibres still reveals traces of PEO and only a low level of protonation of the carboxylate groups of alginate (Figure S5). Therefore, the treated fibres can easily dissolve if 
exposed to aqueous environments. The treatment with formic acid $\left(\mathrm{pK}_{\mathrm{a}}=3.8\right)$ affects the network organisation of the nanofibres, converting the electrospun constructs in uniform thin polymer films. This is more evident with strong acids, like $\mathrm{HCl}$, that even determine the complete dissolution of the mats due to the presence of water and the hydrolysis of alginate ${ }^{32}$. As demonstrated by other studies, the availability of scaffolds constituted by nanometric fibres is of particular interest in regenerative medicine because it positively affects the growth of cells ${ }^{33}$. Furthermore, the produced alginate scaffolds treated by TFA are free from cytotoxic chemicals and possess structural properties that can promote the attachment and proliferation of fibroblast cells, as proved by the biocompatibility analysis in Figure 5. On the contrary, it has been demonstrated that the use of chemical cross-linkers (like GTA) or secondary ions (such as Ca) to improve the stability of alginate can result in a reduction of the biocompatibility of the produced structures, strongly limiting their applicability in the biomedical field. For instance, GTA adversely affects cells proliferation, and consequently the cross-linked materials can exhibit cytotoxicity and induce cell apoptosis ${ }^{19,34}$. On the other hand, it has been shown that high concentrations of $\mathrm{Ca}$ in cell culture media can lead to a loss of cells adhesion and viability ${ }^{35}$. Studies on Ca-alginate wound dressings have also shown cytopathic effects on both fibroblasts and epidermal cells, due to the release of Ca ions ${ }^{36}$. Therefore, the use of the TFA treatment guarantees both enhanced stability and biocompatibility of the alginate structures.

\section{CONCLUSIONS}

In this work, we propose the use of trifluoroacetic acid to tune the biodegradation properties of electrospun alginate nanofibres. Chemical analysis revealed that TFA mainly acted to protonate the carboxylate groups of sodium alginate, forming water insoluble poly(alginic acid). Therefore, 
the resulting nanofibres exhibited an enhanced stability in aqueous environment compared to the initial sodium alginate ones. We observed that by changing the time duration of the TFA-alginate reaction, we modify the biodegradation rate of the fibres. In particular, the fibres treated for $3 \mathrm{~h}$ with TFA degraded in less than 10 days; instead their degradation was extended up to 14 days by using a TFA procedure that lasted 12 or $24 \mathrm{~h}$. In all the cases, the topography of the electrospun mats was preserved. The availability of a procedure able to control the biodegradability of electrospun alginate meshes under physiological condition is attractive for the development of biomedical devices to be used in regenerative medicine and drug delivery.

\section{AUTHOR INFORMATION}

\section{Corresponding Authors}

*hadi.hajiali@iit.it; elisa.mele@iit.it

\section{Author Contributions}

The manuscript was written through contributions of all authors. All authors have given approval to the final version of the manuscript.

\section{ACKNOWLEDGMENTS}

Silvia Dante is acknowledged for providing the NIH/3T3 cells. J. A. Heredia-Guerrero acknowledges the BIOPROTO project (Marie Curie Intra-European Fellowship), financed by the EU Seventh Framework Programme for Research (FP7).

\section{SUPPORTING INFORMATION}


Supporting information associated with this article includes four figures and one table. This material is available free of charge via the Internet at http://pubs.acs.org.

\section{ABBREVIATIONS}

SA, sodium alginate; PEO, polyethylene oxide; TFA, trifluoroacetic acid; $\mathrm{HCl}$, hydrochloric acid; $\mathrm{NaOH}$, sodium hydroxide; DMF, dimethylformamide; DMEM, Dulbecco's Modified Eagle's medium; BCS, bovine calf serum; MTT, thiazolyl blue tetrazolium bromide; PBS, phosphate Buffered Saline.

\section{REFERENCES}

1. Chrzanowski, W.; Khademhosseini, A., Biologically inspired 'smart' materials. Adv. Drug Delivery Rev. 2013, 65, 403-404.

2. Lee, K. Y.; Jeong, L.; Kang, Y. O.; Lee, S. J.; Park, W. H., Electrospinning of polysaccharides for regenerative medicine. Adv. Drug Delivery Rev. 2009, 61, 1020-1032.

3. Li, X.; Chen, S.; Zhang, B.; Li, M.; Diao, K.; Zhang, Z.; Li, J.; Xu, Y.; Wang, X.; Chen, H., In situ injectable nano-composite hydrogel composed of curcumin, N,O-carboxymethyl chitosan and oxidized alginate for wound healing application. Int. J. Pharm. 2012, 437, 110-119.

4. Szarek, D.; Marycz, K.; Bednarz, P.; Tabakow, P.; Jarmundowicz, W.; Laska, J., Influence of calcium alginate on peripheral nerve regeneration: In vivo study. Biotechnol. Appl. Biochem. 2013, 60, 547-556. 
5. Tang, M.; Chen, W.; Weir, M. D.; Thein-Han, W.; Xu, H. H., Human embryonic stem cell encapsulation in alginate microbeads in macroporous calcium phosphate cement for bone tissue engineering. Acta Biomater. 2012, 8, 3436-3445.

6. Wang, C. C.; Yang, K. C.; Lin, K. H.; Liu, Y. L.; Liu, H. C.; Lin, F. H., Cartilage regeneration in SCID mice using a highly organized three-dimensional alginate scaffold. Biomaterials 2012, 33, 120-127.

7. Abbah, S. A.; Liu, J.; Lam, R. W.; Goh, J. C.; Wong, H. K., In vivo bioactivity of rhBMP-2 delivered with novel polyelectrolyte complexation shells assembled on an alginate microbead core template. J. Controlled Release 2012, 162, 364-372.

8. Liakos, I.; Rizzello, L.; Scurr, D. J.; Pompa, P. P.; Bayer, I. S.; Athanassiou, A., Allnatural composite wound dressing films of essential oils encapsulated in sodium alginate with antimicrobial properties. Int. J. Pharm. 2014, 463, 137-145.

9. (a) Mele, E.; Anyfantis, G. C.; Fragouli, D.; Ruffilli, R.; Athanassiou, A., Localized synthesis of gold nanoparticles in anisotropic alginate structures. RSC Adv. 2014, 4, 2044920453; (b) Mele, E.; Fragouli, D.; Ruffilli, R.; De Gregorio, G. L.; Cingolani, R.; Athanassiou, A., Complex architectures formed by alginate drops floating on liquid surfaces. Soft Matter 2013, 9, 6338-6343.

10. Shapiro, L.; Cohen, S., Novel alginate sponges for cell culture and transplantation. Biomaterials 1997, 18, 583-590. 
11. Bonino, C. A.; Efimenko, K.; Jeong, S. I.; Krebs, M. D.; Alsberg, E.; Khan, S. A., Threedimensional electrospun alginate nanofiber mats via tailored charge repulsions. Small 2012, 8, 1928-1936.

12. (a) Hajiali, H.; Mele, E.; Liakos, I.; Bayer, I. S.; Athanassiou, A., Bioactive nanofibrous materials for wound healing. J. Tissue Eng. Regener. Med. 2014, 8, 504-505; (b) Hajiali, H.; Shahgasempour, S.; Naimi-Jamal, M. R.; Peirovi, H., Electrospun PGA/gelatin nanofibrous scaffolds and their potential application in vascular tissue engineering. Int. J. Nanomed. 2011, 6, 2133-2141.

13. Sill, T. J.; von Recum, H. A., Electrospinning: Applications in drug delivery and tissue engineering. Biomaterials 2008, 29, 1989-2006.

14. Yildirimer, L.; Seifalian, A. M., Three-dimensional biomaterial degradation - Material choice, design and extrinsic factor considerations. Biotechnol. Adv. 2014, 32, 984-999.

15. Narayan, B.; Miqin, Z., Controlled synthesis and structural stability of alginate-based nanofibers. Nanotechnology 2007, 18, 455601.

16. (a) Kim, Y. J.; Yoon, K. J.; Ko, S. W., Preparation and properties of alginate superabsorbent filament fibers crosslinked with glutaraldehyde. J. Appl. Polym. Sci. 2000, 78, 1797-1804; (b) Kulkarni, A. R.; Soppimath, K. S.; Aminabhavi, T. M.; Dave, A. M.; Mehta, M. H., Glutaraldehyde crosslinked sodium alginate beads containing liquid pesticide for soil application. J. Controlled Release 2000, 63, 97-105; (c) Kulkarni, A. R.; Soppimath, K. S.; Aralaguppi, M. I.; Aminabhavi, T. M.; Rudzinski, W. E., Preparation of cross-linked sodium alginate microparticles using glutaraldehyde in methanol. Drug Dev. Ind. Pharm. 2000, 26, $1121-1124$. 
17. (a) Augst, A. D.; Kong, H. J.; Mooney, D. J., Alginate hydrogels as biomaterials. Macromol. Biosci. 2006, 6, 623-633; (b) Gopishetty, V.; Tokarev, I.; Minko, S., Biocompatible stimuli-responsive hydrogel porous membranes via phase separation of a polyvinyl alcohol and Na-alginate intermolecular complex. J. Mater. Chem. 2012, 22, 19482-19487; (c) Kuo, C. K.; Ma, P. X., Ionically crosslinked alginate hydrogels as scaffolds for tissue engineering: part 1. Structure, gelation rate and mechanical properties. Biomaterials 2001, 22, 511-521; (d) Ma, G.; Fang, D.; Liu, Y.; Zhu, X.; Nie, J., Electrospun sodium alginate/poly(ethylene oxide) core-shell nanofibers scaffolds potential for tissue engineering applications. Carbohydr. Polym. 2012, 87, 737-743.

18. Hennink, W. E.; van Nostrum, C. F., Novel crosslinking methods to design hydrogels. Adv. Drug Delivery Rev. 2002, 54, 13-36.

19. (a) Lai, J.-Y., Biocompatibility of Genipin and Glutaraldehyde Cross-Linked Chitosan Materials in the Anterior Chamber of the Eye. Int. J. Mol. Sci. 2012, 13, 10970-10985; (b) Lai, J. Y., Biocompatibility of chemically cross-linked gelatin hydrogels for ophthalmic use. J. Mater. Sci.: Mater. Med. 2010, 21, 1899-1911.

20. Jeong, S. I.; Krebs, M. D.; Bonino, C. A.; Khan, S. A.; Alsberg, E., Electrospun alginate nanofibers with controlled cell adhesion for tissue engineering. Macromol. Biosci. 2010, 10, 934943.

21. Stijnman, A. C.; Bodnar, I.; Hans Tromp, R., Electrospinning of food-grade polysaccharides. Food Hydrocolloids 2011, 25, 1393-1398. 
22. Hajiali, H.; Hosseinalipour, M.; Karbasi, S.; Shokrgozar, M. A., The influence of bioglass nanoparticles on the biodegradation and biocompatibility of poly (3-hydroxybutyrate) scaffolds. Int. J. Artif. Organs 2012, 35, 1015-1024.

23. Yoshihara, T.; Tadokoro, H.; Murahashi, S., Normal Vibrations of the Polymer Molecules of Helical Conformation. IV. Polyethylene Oxide and Polyethylene-d4 Oxide. J. Chem. Phys. 1964, 41, 2902-2911.

24. (a) Jejurikar, A.; Seow, X. T.; Lawrie, G.; Martin, D.; Jayakrishnan, A.; Grondahl, L., Degradable alginate hydrogels crosslinked by the macromolecular crosslinker alginate dialdehyde. J. Mater. Chem. 2012, 22, 9751-9758; (b) Lawrie, G.; Keen, I.; Drew, B.; ChandlerTemple, A.; Rintoul, L.; Fredericks, P.; Grøndahl, L., Interactions between Alginate and Chitosan Biopolymers Characterized Using FTIR and XPS. Biomacromolecules 2007, 8, 25332541.

25. (a) Gómez-Ordóñez, E.; Rupérez, P., FTIR-ATR spectroscopy as a tool for polysaccharide identification in edible brown and red seaweeds. Food Hydrocolloids 2011, 25, 1514-1520; (b) Mackie, W., Semi-quantitative estimation of the composition of alginates by infra-red spectroscopy. Carbohydr. Res. 1971, 20, 413-415.

26. Lu, J.-W.; Zhu, Y.-L.; Guo, Z.-X.; Hu, P.; Yu, J., Electrospinning of sodium alginate with poly(ethylene oxide). Polymer 2006, 47, 8026-8031.

27. (a) CRC Handbook of Chemistry and Physics, 87th Edition (CRC Handbook of Chemistry \& Physics). CRC Press: Boca Raton, 2006; (b) Donati, I.; Paoletti, S., Material properties of alginates. In Alginates: biology and applications, Springer: 2009; pp 1-53. 
28. Smidsrød, O.; Larsen, B. R.; Painter, T.; Haug, A., The role of intramolecular autocatalysis in the acid hydrolysis of polysaccharides containing 1, 4-linked hexuronic acid. Acta Chem. Scand. 1969, 23, 1573-1580.

29. (a) Haug, A.; Larsen, B., The solubility of alginate at low pH. Acta Chem. Scand. 1963, 17, 1653-1662; (b) Huang, R. Y. M.; Pal, R.; Moon, G. Y., Characteristics of sodium alginate membranes for the pervaporation dehydration of ethanol-water and isopropanol-water mixtures. J. Membr. Sci. 1999, 160, 101-113.

30. Hirai, A.; Odani, H., Sorption and transport of water vapor in alginic acid, sodium alginate, and alginate-cobalt complex films. J. Polym. Sci., Part B: Polym. Phys. 1994, 32, 23292337.

31. Chang, J.-J.; Lee, Y.-H.; Wu, M.-H.; Yang, M.-C.; Chien, C.-T., Preparation of electrospun alginate fibers with chitosan sheath. Carbohyr. Polym. 2012, 87, 2357-2361.

32. Inoue, A.; Takadono, K.; Nishiyama, R.; Tajima, K.; Kobayashi, T.; Ojima, T., Characterization of an alginate lyase, FlAlyA, from Flavobacterium sp. strain UMI-01 and its expression in Escherichia coli. Marine drugs 2014, 12, 4693-712.

33. Hsia, H. C.; Nair, M. R.; Mintz, R. C.; Corbett, S. A., The fiber diameter of synthetic bioresorbable extracellular matrix influences human fibroblast morphology and fibronectin matrix assembly. Plast. Reconstr. Surg. 2011, 127, 2312-2320.

34. Sung, H. W.; Huang, R. N.; Huang, L. L.; Tsai, C. C., In vitro evaluation of cytotoxicity of a naturally occurring cross-linking reagent for biological tissue fixation. J. Biomater. Sci., Polym. Ed. 1999, 10, 63-78. 
35. Rowley, J. A.; Sun, Z.; Goldman, D.; Mooney, D. J., Biomaterials to Spatially Regulate Cell Fate. Adv. Mater. 2002, 14, 886-889.

36. (a) Suzuki, Y.; Nishimura, Y.; Tanihara, M.; Suzuki, K.; Nakamura, T.; Shimizu, Y.; Yamawaki, Y.; Kakimaru, Y., Evaluation of a novel alginate gel dressing: Cytotoxicity to fibroblasts in vitro and foreign-body reaction in pig skin in vivo. J. Biomed. Mater. Res. 1998, 39, 317-322; (b) Stenvik, J.; Sletta, H.; Grimstad, Ø.; Pukstad, B.; Ryan, L.; Aune, R.; Strand, W.; Tøndervik, A.; Helge Torp, S.; Skjåk-Bræk, G.; Espevik, T., Alginates induce differentiation and expression of CXCR7 and CXCL12/SDF-1 in human keratinocytes-The role of calcium. $J$. Biomed. Mater. Res., Part A 2012, 100, 2803-2812.

\section{FIGURES}
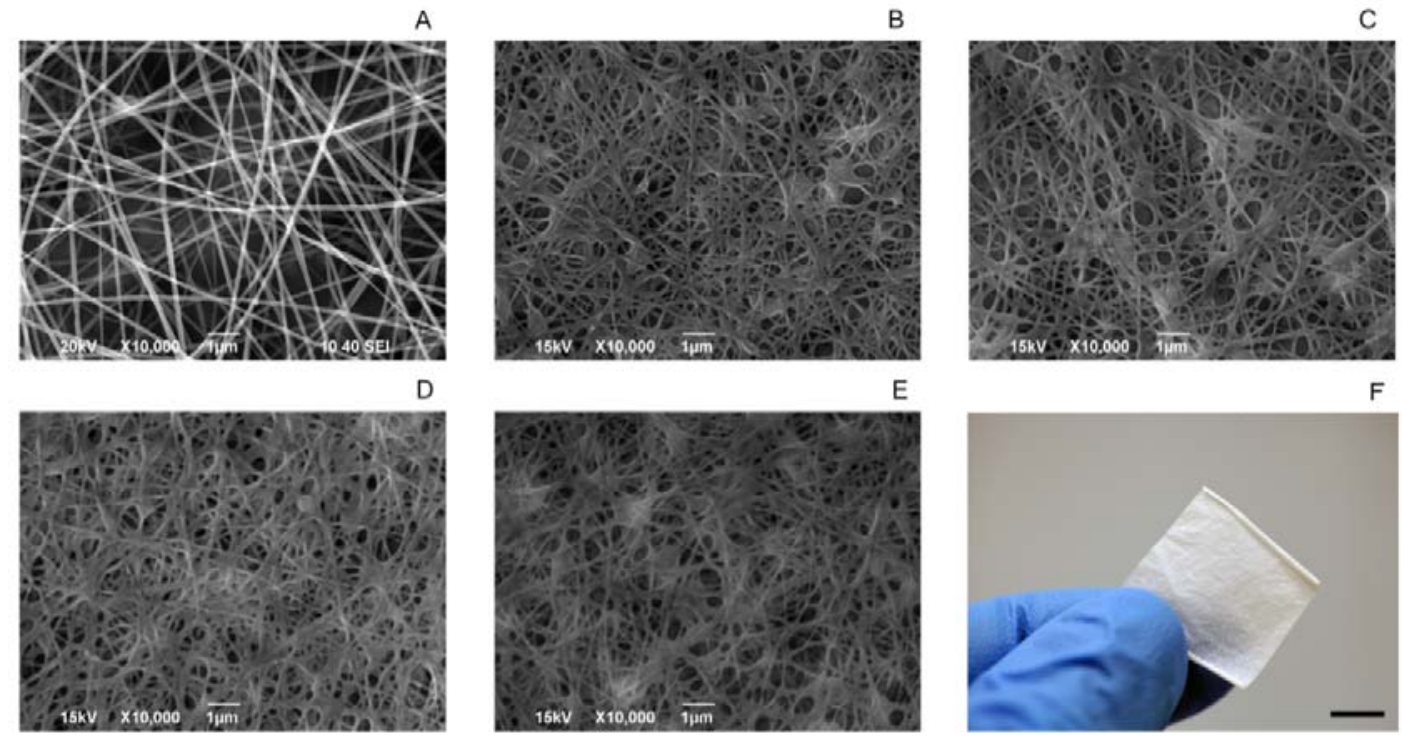
Figure 1. SEM images of the electrospun alginate nanofibres: (A) as-prepared, and after the TFA treatment for (B) $3 \mathrm{~h}$, (C) $6 \mathrm{~h},(\mathbf{D}) 12 \mathrm{~h}$, and (E) $24 \mathrm{~h}$. (F) Photograph of the nanofibrous scaffolds after $24 \mathrm{~h}$ of the acid procedure. Scale bar $=0.5 \mathrm{~cm}$.
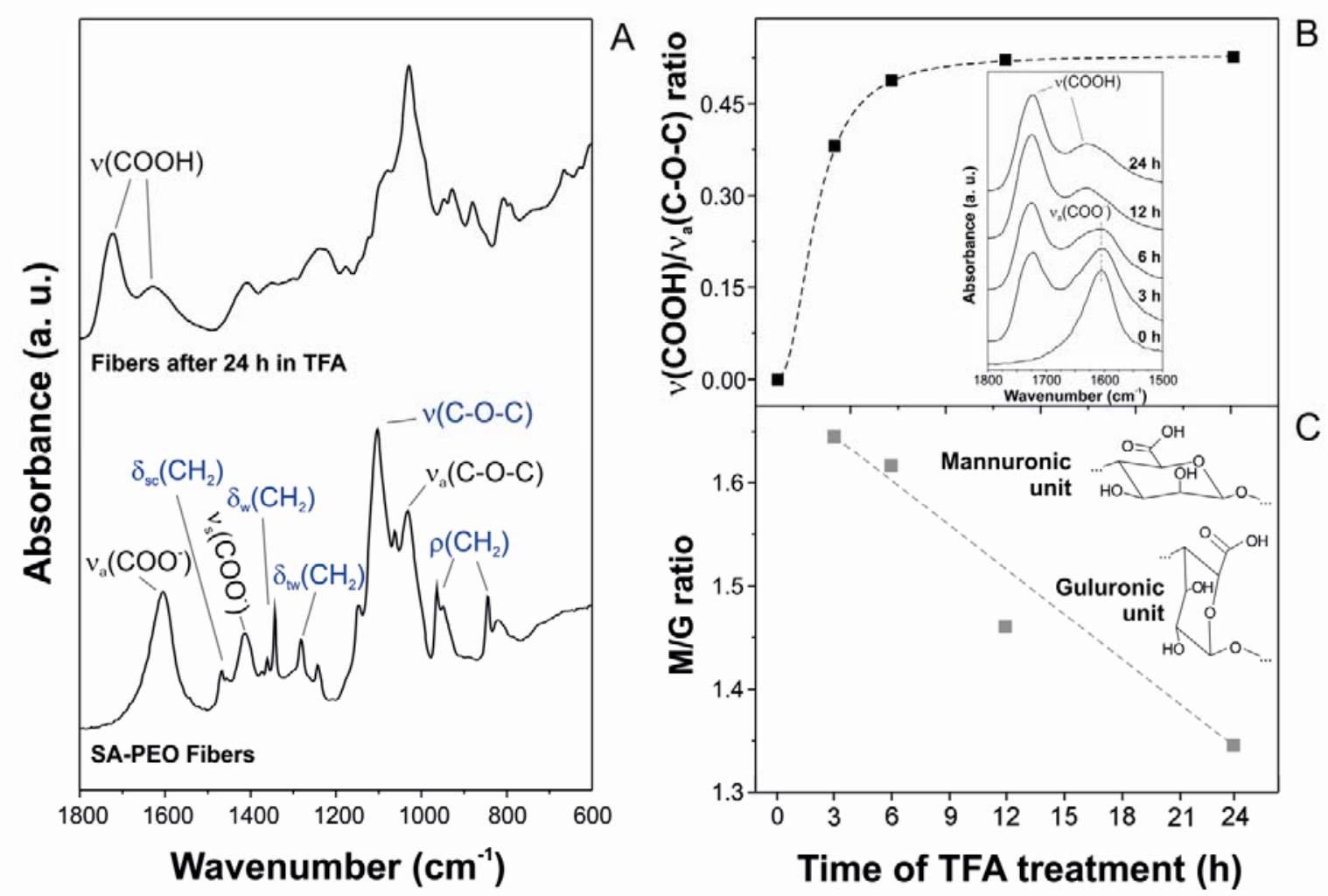
Figure 2. (A) ATR-FTIR spectra of SA-PEO fibres before and after the TFA treatment $(24 \mathrm{~h})$. The assignment of bands ascribed to PEO is highlighted in blue, while the corresponding one for SA is in black. (B) $v(\mathrm{COOH}) / v_{\mathrm{a}}(\mathrm{C}-\mathrm{O}-\mathrm{C})$ ratio as a function of the duration of the TFA treatment. Inset: spectral region associated to the stretching vibrations of $-\mathrm{C} \mid \mathrm{OOH}$ and $-\mathrm{COO}^{-}$groups. (C) Variation of the mannuronic/guluronic $(\mathrm{M} / \mathrm{G})$ ratio of the fibres with the time of treatment in TFA. Inset: chemical structure of the M and G units.

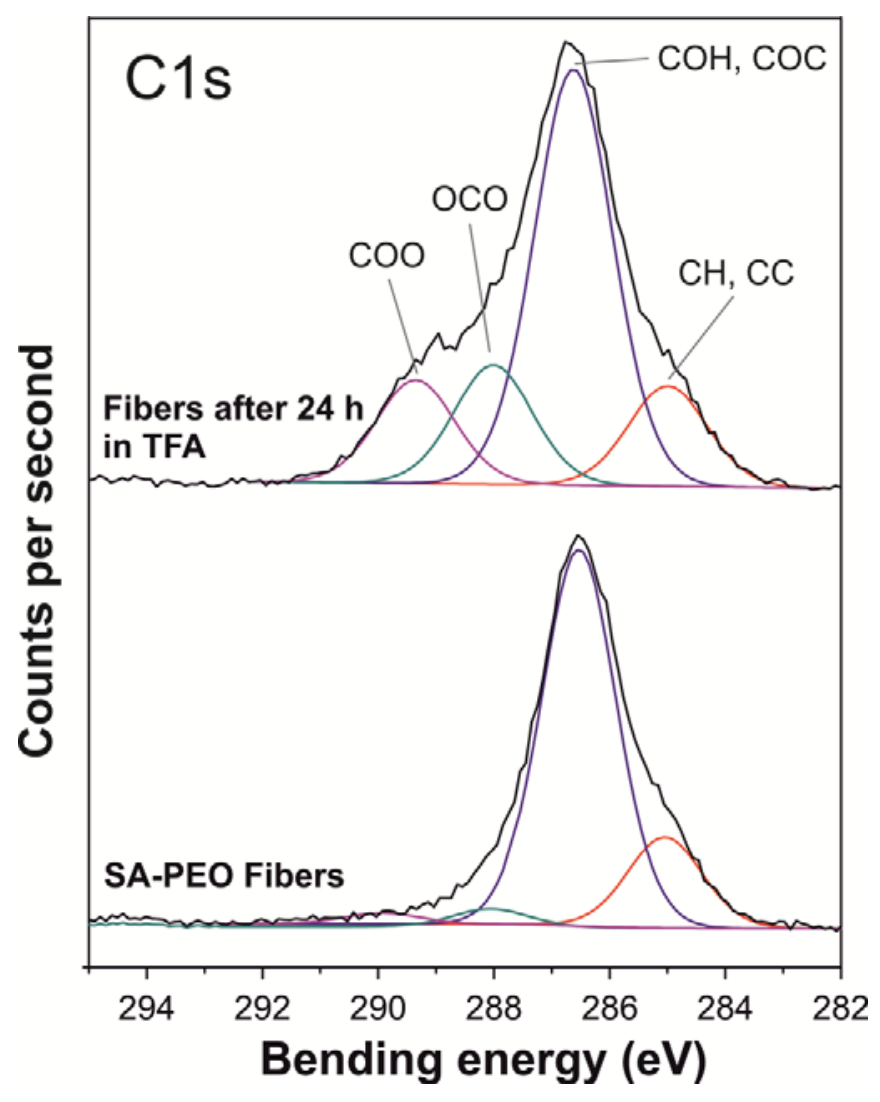


Figure 3. C1s core-level spectra of XPS for the alginate nanofibres before and after the TFA treatment (24h).

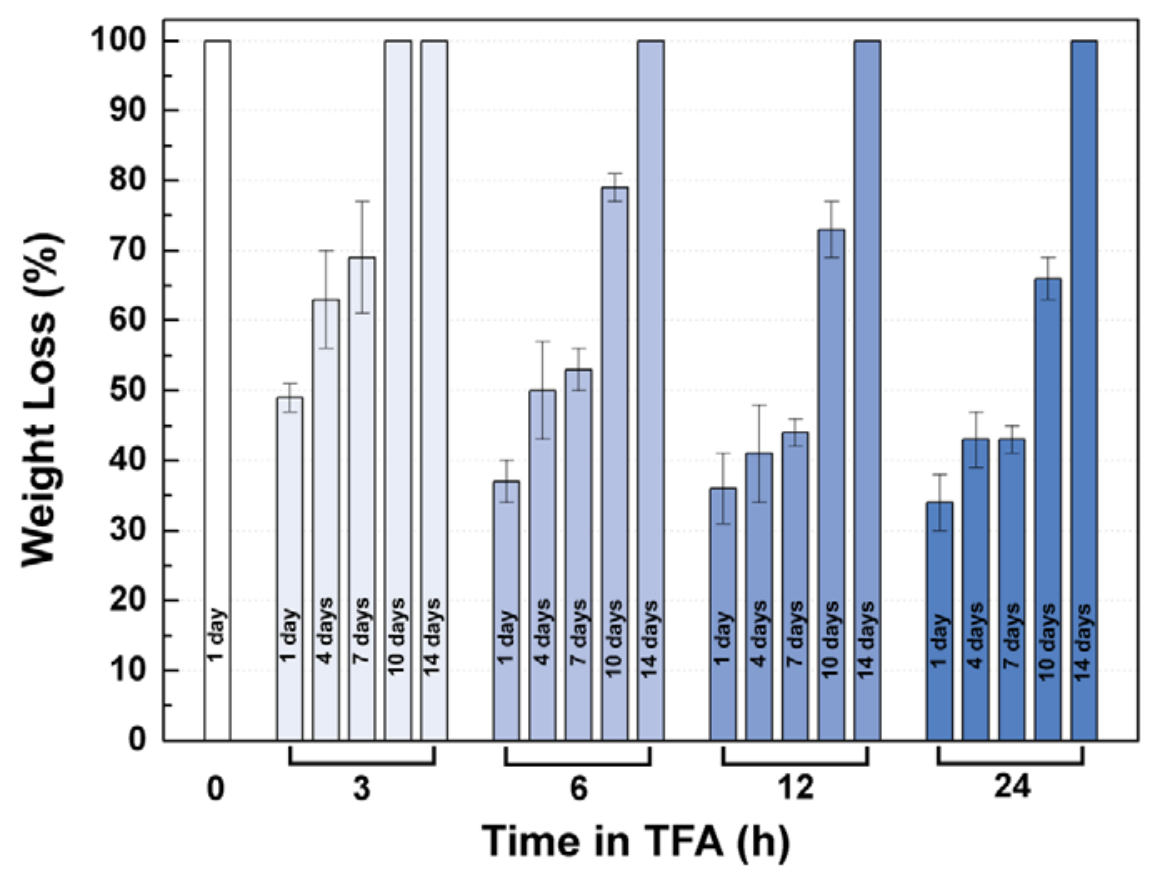


Figure 4. Analysis of the degradation properties of the alginate nanofibres before and after the TFA procedure at different time intervals $(3,6,12$ and $24 \mathrm{~h})$. The weight loss of the samples was measured after $1,4,7,10$ and 14 days of PBS incubation under physiological conditions $\left(37^{\circ} \mathrm{C}\right.$, $\mathrm{pH} 7.4)$. 

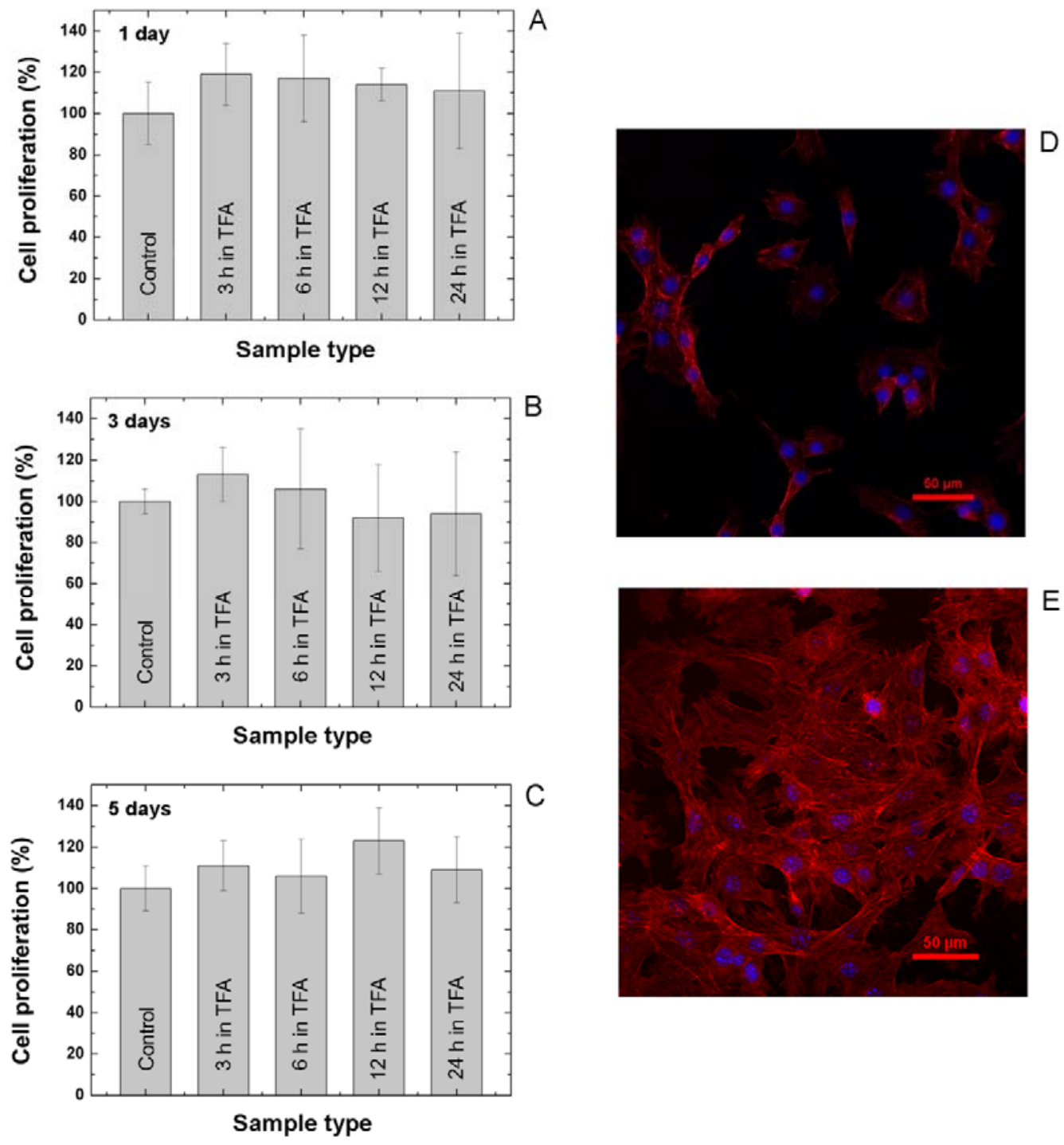

Figure 5. Viability of fibroblast cells (MTT assay) on the different types of treated nanofibrous constructs after (A) 1, (B) 3 and (C) 5 days. Fluorescence micrographs of DAPI (nucleus) and phalloidin (actin filaments) stained fibroblast cells, cultured on (D) control (glass substrate), and (E) treated nanofibres (24 h in TFA). 


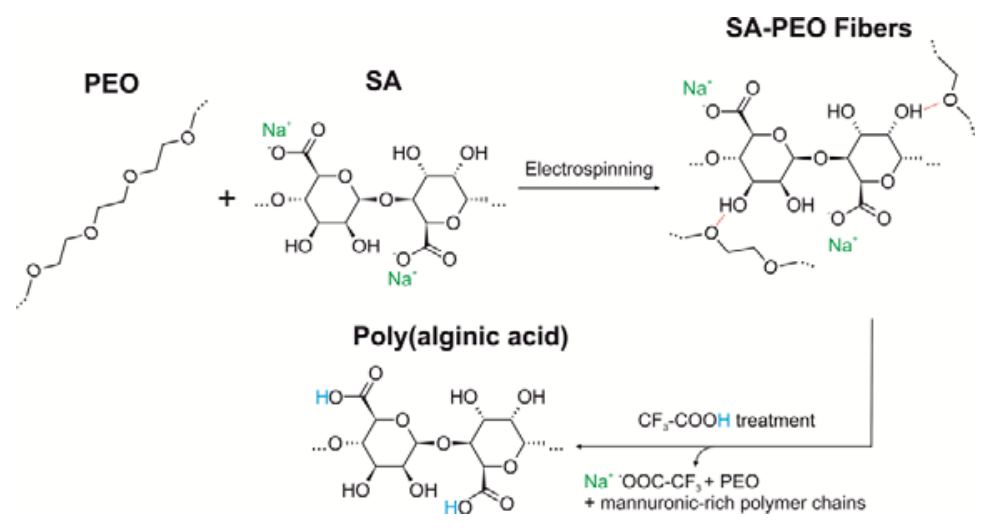

Figure 6. Schematic representation of the chemical modifications of the alginate molecular structure during the TFA treatment. 